\title{
Tangence
}

\section{Américanité et antropophagie littéraire dans Monsieur Melville}

Jean Morency

Numéro 41, octobre 1993

Interdiscurtivité dans l'œuvre de Victor-Lévy Beaulieu

URI : https://id.erudit.org/iderudit/025777ar

DOI : https://doi.org/10.7202/025777ar

Aller au sommaire du numéro

Éditeur(s)

Tangence

ISSN

0226-9554 (imprimé)

1710-0305 (numérique)

Découvrir la revue

Citer cet article

Morency, J. (1993). Américanité et antropophagie littéraire dans Monsieur

Melville. Tangence, (41), 54-68. https://doi.org/10.7202/025777ar d'utilisation que vous pouvez consulter en ligne.

https://apropos.erudit.org/fr/usagers/politique-dutilisation/ 


\title{
Américanité et anthropophagie littéraire dans Monsieur Melville
}

\author{
Jean Morency*
}

Dans les premières pages de son "Journal" de 1964, VictorLévy Beaulieu mentionne l'histoire pour le moins étonnante de Léonard Euler, "un très grand mathématicien du XvII ${ }^{\mathrm{e}}$ siècle qui était capable de réciter entièrement et sans erreur tous les livres qu'il avait lus dans sa vie" ${ }^{1}$. Dans ces quelques lignes, on retrouve déjà exprimée avant la lettre (Beaulieu est âgé alors de dix-neuf ans et n'a pas encore entrepris la rédaction de son œuvre), l'ambition secrète de l'auteur de La vraie saga des Beaucbemin, soit celle de pouvoir, non pas vraiment réciter, entièrement et sans erreur, tous les livres importants de sa vie, mais bien de les intégrer à son ouvre littéraire. Vaste entreprise que celle-ci, on en conviendra, qui se situe à la croisée de la lecture et de l'écriture, au carrefour des discours, et qui vise à restituer, dans toute leur beauté et leur vérité, certains grands textes fondateurs, mais sans qu'ils ressortent dénaturés pour autant du creuset de la création romanesque.

On connaît assez bien les écrivains de prédilection de Beaulieu, non seulement par l'entremise des essais qu'il a consacrés à Hugo, Kerouac, Melville, Ferron et Tolstoï, mais aussi par les nombreuses références qui sont faites, dans ses romans, à Homère, Cervantes, Flaubert ou Joyce. À l'intérieur de ce panthéon littéraire, les écrivains américains - outre Melville et Kerouac, citons Faulkner et Burroughs, et aussi Hawthorne occupent une place si importante qu'un critique comme Jonathan M. Weiss est allé jusqu'à affirmer, pour la forme, que Beaulieu était un écrivain américain! Selon Weiss, l'“américanité " des romans de Beaulieu est liée, d'une part, aux influences littéraires de l'écrivain: "Lecteur vorace [...], Beaulieu retient certains écrivains avec qui il établit un rapport presque gémellaire, et ces écrivains,

Jean Morency est membre du Centre de recherche en littérature québécoise (Université Laval) et professeur à l'Université Trent.

1 Victor-Lévy Beaulieu, Entre la sainteté et le terrorisme, Montréal, VLB éditeur, 1984, p. 26. 
hormis Hugo, sont surtout américains" 2 , et d'autre part, à une forme de parenté imaginaire qui relie l'univers romanesque de Beaulieu aux thèmes et aux structures caractérisant la littérature classique américaine, tels que dévoilés par des critiques comme Leslie Fiedler et Richard Slotkin.

La question de l'américanité de la littérature québécoise pose plusieurs problèmes d'ordre méthodologique. Le concept luimême n'est pas encore clairement défini. On confond encore trop souvent américanité et étatsunicité, limitant du même coup la portée opératoire du concept à des questions d'influence littéraire ou culturelle, ou aux références qui y sont faites, à l'intérieur du corpus littéraire québécois, aux États-Unis et aux paysages américains. Il s'agit en fait d'une notion encore assez vague qui ne sert parfois qu'à masquer une carence de rigueur intellectuelle, ou qui ne constitue, selon François Ricard, grand pourfendeur du concept en question, "qu'un nouvel avatar du besoin de spécificité, une nouvelle manifestation de la tentation qu'éprouvent plus ou moins tous les provinciaux de se montrer "pittoresques", en se définissant par cela même qui fait d'eux des provinciaux" 3 . Il faut néanmoins avouer que certaines tentatives très intéressantes de mieux asseoir le concept en élargissant sa portée ont été accomplies au cours des dernières années, comme en témoignent les études de plus en plus nombreuses de littérature comparée consacrées aux rapports entre le Québec et les États-Unis, et aussi entre le Québec et l'Amérique latine ${ }^{4}$, ou encore les travaux de Jean-François Chassay visant à articuler le concept d'américanité autour de l'idéologie de la communication, qui a pris naissance, en Amérique du Nord, dans une société "sans mémoire" 5 , pour reprendre l'expression de Lucien Sfez.

2 Jonathan M. Weiss, "Victor-Lévy Beaulieu: écrivain américain", Études françaises, vol. XIX, $\mathrm{n}^{\circ} 1$, printemps 1983 , p. 42.

3 François Ricard, "Remarques sur la normalisation d'une littérature", Ecriture (Lausanne), $\mathrm{n}^{\circ} 31$, automne 1988, p. 16.

4 Consulter les articles parus dans les revues suivantes: Voix et images, n 34 , automne 1986, "Dossier comparatiste Québec-Amérique latine"; Etudes françaises, vol. XXVI, $\mathrm{n}^{\circ} 2$, automne 1990, "L'Amérique de la littêrature québécoise ; Urgences, no 34, décembre 1991, "Mythes et romans de l'Amérique"; Études françaises, vol. XXVIII, $\mathrm{n}^{\text {os }} 2-3$, automne 1992-hiver 1993, "L'Amérique entre les langues".

5 Jean-François Chassay, "Reflet des États-Unis dans le roman québécois: une version de l'Amérique ", Urgences, $\mathrm{n}^{\circ}$ 34, décembre 1991, p. 7-19. 
Parmi les nouvelles avenues de recherche proposées, l'une des plus fécondes me semble être celle de l'anthropophagie littéraire. Avant de tirer au clair cette notion, autant que faire se peut, je voudrais remarquer que généralement, on va associer l'américanité des littératures américaines à la volonté des créateurs et des institutions de rompre avec les instances de légitimation et les modèles littéraires européens. Je citerai ici l'exemple de Moby Dick de Herman Melville et de The Scarlet Letter de Nathaniel Hawthorne, deux romans qui sont parus au début des années 1850 et qui marquent la naissance d'une littérature véritablement étatsunienne, qui se voulait enfin affranchie de la sphère culturelle anglaise. Cette volonté de rupture caractérisant l'américanité s'exprimerait par conséquent, au niveau des institutions, par l'affirmation des différentes littératures nationales; au niveau des formes et des techniques, par la quête de la modernité, de la rupture et de la différence; et sur le plan des contenus, par le désir de cristalliser la réalité américaine, qu'il s'agisse de son espace, de son histoire ou de ses mythes. Paradoxalement, au cour même de la rupture qui la définit, l'américanité apparaît donc comme étant indissociable de ses sources européennes, en l'occurrence, au Québec, de la "francité ".

C'est ici qu'entre en ligne de compte la notion d'anthropophagie, proposée en 1928 par Oswald de Andrade, un des représentants du modernisme brésilien. Dans son Manifeste antbropophage, de Andrade, s'inspirant de la métaphore de l'Indien cannibale, renverse les données du problème des littératures nationales américaines: selon lui, les peuples colonisés doivent dévorer l'autre (le colonisateur) et assimiler sa culture pour ne pas être dévorés par lui. Il ne préconise donc non pas tant une rupture qu'un dépassement, comme le mentionne la critique brésilienne Leyla Perrone-Moisés: "Oswald de Andrade, comme tous les modernistes américains, pose la question de l'identité nationale; cependant, à la différence de la plupart des Américains, cette identité n'est pas posée par lui comme refus de l'autre (l'Européen, le colonisateur), mais comme absorption de l'autre $*$. Or, cette notion de l'anthropophagie n'a été que rare-

6 Leyla Perrone-Moisés, "Littérature comparée, intertexte et anthropophagie", dans Confluences littéraires. Brêsil-Québec: les bases d'une comparaison, sous la direction de Michel Peterson et Zila Bernd, Candiac, Éditions Balzac, coll. "L'Univers des discours", 1992, p. 182. 
ment associée à l'étude du corpus québécois. Pourtant, selon Benoît Melançon, qui s'est intéressé aux perspectives de recherche offertes par le concept d'américanité,

Malgré les nuances qu'il faudrait apporter à cette métaphore [celle de l'anthropophagiel pour l'appliquer au domaine québécois, elle pourrait permettre de relancer un débat qui, bien que soit réelle la diversité des contributions, manque souvent de renouvellement. La notion de dévoration culturelle (de la France, de l'Amérique) pourrait être une de ces sources de renouvellement ${ }^{7}$.

Cette dévoration culturelle dont parle Melançon semble être au cœur de l'œuvre de Victor-Lévy Beaulieu, ce "lecteur vorace", pour reprendre les mots de Jonathan $M$. Weiss. Déjà, dans un texte paru dans Le devoir en 1973, au moment de la publication des Recréants de Jean-Marie Poupart, un ouvrage qui me semble devoir être considéré comme ayant contribué à la généalogie de Monsieur Melville, Beaulieu affirmait:

Poupart a assimilé le roman policier à son monde, de sorte que lorsqu'il en parle il ne le fait pas de l'extérieur ni de haut mais de l'intérieur, brillamment, en mêlant avec harmonie des bribes de son propre roman à son cuvre d'essayiste. ${ }^{8}$

Les recréants annoncerait, en quelque sorte, le geste anthropophage qui guidera Beaulieu, quatre ans plus tard, dans son exploration et son assimilation de l'univers melvillien. Toujours à propos des Recréants, Beaulieu ajoutait en effet:

Mais ce qui me paraît important dans ce nouveau genre d'expérience à la Poupart, c'est le fait que pour la première fois des auteurs étrangers nous sont présentés d'un point de vue québécois, dans l'éclairage de ce qui constitue notre originalité. À ce point de vue encore, nous sommes dans une position privilégiée, notre situation exceptionnelle sur le continent américain faisant naturellement de nous des observateurs attentifs à tout ce qui se passe dans le monde. De la sorte, nous pouvons profiter de toutes les expériences étrangères et les refondre, à notre avantage, dans notre propre projet collectif."

Cette refonte des expériences étrangères ne va pas sans rappeler les idées défendues pas Oswald de Andrade dans son Manifeste

7 Benoît Melançon, "Lá littérature québécoise et l'Amérique. Prolégomènes et bibliographie", Études françaises, vol. XXVI, $\mathrm{r}^{\circ} 2$, automne 1990 , p. 80.

8 Beaulieu, op. cit., p. 225.

9 Ibid. 
anthropophage. Tout se passe comme si certains écrivains québécois, dans les années 1970, se trouvaient sensibilisés à la problématique envisagée, cinquante ans plus tôt, par les modernistes brésiliens. Je ne crois pas que l'on doive se formaliser de ce décalage temporel, mais y voir plutôt l'indice de la permanence du rapport problématique que les Québécois, citoyens d'un "pays incertain", pour reprendre l'expression de Jacques Ferron, entretiennent avec l'Europe et l'Amérique. Dans un autre texte, "L'écrivain québécois et le pays équivoque", paru peu avant l'élection du Parti québécois en 1976, Beaulieu se prononcera encore plus clairement sur le problème de la dévoration culturelle:

C'est qu'écrire n'est rien de moins que du pillage et il est important de prendre à l'autre son butin, ne serait-ce que pour se revêtir de ses mots et pour s'armer de leur puissance. On en avait besoin et l'autre en avait besoin aussi. De toute façon, cela faisait partie du projet que, secrètement, on entretenait en soi: tout dire, de toutes les façons possibles, en se servant de tous les mots imaginables, sans pitié et sans pudeur, rien que pour jouir de sa liberté et parce qu'on acceptait difficilement l'admiration, se considérant plutôt comme l'égal de n'importe quel créateur vivant ou ayant vécu. On pouvait et on était Joyce, Rabelais, Melville, Dante, Broch, Marquez, Asturias, Homère, Hugo, Cervantes et Ferron. On pouvait et on était tous ces créateurs en même temps parce qu'on en avait décidé ainsi, après étude du pays équivoque qui vous avait amené à cette conclusion: loin d'être un empêchement, le pays équivoque constituait votre force parce qu'il flottait dans le non-espace, faisant de vous un médium, capable de tout, même de ne pas être et pourtant d'y être parce que justement, on n'y était pas. ${ }^{10}$

L'écrivain du pays équivoque devient ainsi le réceptacle de tous les discours, de tous les textes, de tous les mots. Il se tient au milieu d'une multitude de langages étrangers, dans un espace absent et un temps aboli parce que privés du support d'un projet national clairement établi. L'écrivain du pays équivoque n'existe finalement que dans les mots qu'il dévore inlassablement, dans une vacance géographique et historique parfaite:

Dans le pays équivoque, on peut tout assumer sans vraiment s'intégrer à rien: c'est que l'on y peut tout refuser, c'est que l'on y peut rester invisible par devers les autres. Voilà la chance que l'on a: ne pas participer au vieux monde, n'y pas gaspiller ses

$10 \quad$ Ibid., p. 366-367. 
énergies, mais les rassembler pour préparer ce qui deviendra le nouvel homme, dans l'au-delà de la mort et de la vieille vie. ${ }^{11}$

On peut maintenant constater de quelle façon les concepts d'américanité et d'anthropophagie s'articulent l'un à l'autre. En plaidant pour la venue du "nouvel homme" qui ne participera pas au monde ancien, Beaulieu touche de près au mythe américain du renouvellement et de la métamorphose qui s'avère l'un des mythes importants, sinon le mythe majeur, du Nouveau Monde. Mais ce mythe consacrant la rupture n'exclut pas, chez Beaulieu, l'intégration de toutes les influences possibles, qu'elles soient européennes ou américaines, puisque le pays équivoque se situe quelque part entre les deux continents. Or, cette intégration ne s'effectue jamais de façon occulte, comme le voudrait la vieille théorie des influences littéraires, mais de manière on ne peut plus claire, et c'est ici qu'on rejoint les notions d'intertextualité et d'interdiscursivité, qui me semblent indissociables de la question de la dévoration culturelle. Comme l'observe Laurent Jenny, "[l]'intertextualité désigne non pas une addition confuse et mystérieuse d'influences, mais le travail de transformation et d'assimilation de plusieurs textes opéré par un texte centreur qui garde le leadership du sens " ${ }^{12}$.

Cette problématique du croisement des discours et des textes se trouve d'ailleurs au centre de l'entreprise littéraire de Beaulieu, et cela depuis le commencement de son œuvre. Toujours dans son "Journal", Beaulieu écrivait que "toute ouvre [n'est] que collective, même dans le refus qu'elle pourrait signifier parce qu'elle ne semble être que l'œuvre d'un seul "13. Dès le départ, Beaulieu avait pressenti que son ouvre littéraire se devait de constituer la mise en forme d'un vaste intertexte découlant de l'acte de lecture, intertexte qui se trouvera justement dynamisé par l'exigence de ses sources et de ses modèles:

Mais c'est aussi une prodigieuse vis sans fin, quelque brûlante folie qui, pour occuper tout le centre de ce que vous êtes, vous fait courir de tous bords et de tous côtés, vous fait interroger la culture du monde dans lequel vous vivez, vous force à vous définir dans ce que vous croyez être mais n'êtes pas peut-être car comment penser que l'on pourrait représenter pour son temps ce que jadis

11 Ibid., p. 372.

12 Laurent Jenny, "La stratégie de la forme", Poétique, no 27, 1976, p. 262.

13 Beaulieu, op. cit., p. 15. 
60

Homère, Cervantes, Hugo, Melville, Broch, Joyce, Gaddis; Marquez, Lowry et quelques autres ont été pour le leur? ${ }^{14}$

Cette vaste réflexion intertextuelle, qui fonde en quelque sorte l'œuvre de Beaulieu, remontera à la surface dans N'évoque plus que le désenchantement de ta ténèbre, mon si pauvre Abel, le deuxième volume des Voyageries, une "lamentation" qu'on doit considérer non seulement comme l'avant-texte immédiat de Monsieur Melville, mais aussi comme un traité sur l'écriture qui annonce et définit l'essentiel de son esthétique. Constatant que "[t]oute écriture ne fait jamais que creuser le lit d'écritures autres "15, Abel Beauchemin avoue:

C'est qu'à moi-même je constitue un collectif d'auteurs, prêt à toutes les extravagances, même celles de ma vie, pour faire venir l'œuvte et la totalité de ses images, comme un caméléon paresseux avec ses grands yeux ouverts pour boire le soleil. Ce n'est décidément pas pour rien que dans la ville j'exerce ma fonction d'éditeur: tous ces livres que je fais publier, loin d'empêcher le mien, le poussent à l'avant en accélêrant le temps de sa gestation [..... ${ }^{16}$

Il va même jusqu'à prendre pour modèle William Faulkner, selon qui "le seul écrivain véritable est celui qui a un projet si vaste qu'il ne peut plus le perdre de vue [...] pillant partout, dans les livres comme dans la vie, pour produire l'œuvre abolissant toutes les autres parce que, d'un seul coup, donnant tous leurs sens aux autres" 17. Voici exprimé le désir qui anime Beaulieu au moment de commencer la rédaction de Monsieur Melville. Je voudrais maintenant analyser certains des procédés par lesquels s'expriment l'anthropophagie et l'américanité dans ce livre qu'on peut considérer comme le sommet des romans de Beaulieu, sinon de son ouvre tout entière.

\section{***}

Le premier procédé que je voudrais mettre en lumière est de nature métaphorique. Il s'agit, on l'aura deviné, du processus de

14 Ibid., p. 18.

15 Victor-Lévy Beaulieu, N'évoque plus que le désenchantement de ta ténèbre, mon si pauure Abel, Montréal, VLB éditeur, 1976, p. 111.

16 Ibid., p. 71-72.

17 Ibid., p. 142. 
dévoration et d'assimilation. On sait qu'au tout début du roman, le romancier Abel Beauchemin décide de donner congé à ses personnages pour s'enfermer dans la maison de son père, dans le but d'y écrire son essai sur Melville. Il espère ainsi faire venir à lui l'écrivain américain, non pas pour en faire un personnage de roman, mais pour tenter de comprendre toute la grandeur de l'échec que Melville incarne en tant qu'écrivain. Or, Abel est "convaincu qu'on ne peut pas vraiment rendre compte de la vie d'un homme" 18 et que "[1]a biographie ne peut pas dire ce que Melville a été. (I, 23). D'où la nécessité, pour Abel, de s'approprier Melville en le projetant dans l'univers de sa propre fiction, celle-ci étant libérée, pour l'instant, de ses personnages: "C'est pourquoi Melville, tout comme moi, ne peut que basculer dans l'univers de la fiction" (I, 23), écrit le romancier. Cette appropriation fictive est la première manifestation de la dévoration à laquelle va bientôt se livrer tout entier Abel Beauchemin, qui se compare à un "médium mangeant de l'espace et du temps pour s'approprier le créateur de Moby Dick" (I, 123) et qui avoue par ailleurs: “Écrire, ce n'est pas très difficile, surtout quand je suis comme je suis, capable d'absorber n'importe quoi, le ridicule et le sublime, le dénaturé et le dérisoire" (I, 20). Les métaphores anthropophages réapparaissent ici et là dans Monsieur Melville; tantôt Abel mentionne "cette écriture qu'assis devant la table de pommier [il] mange pour recharger ses batteries" (II, 58), tantôt il avoue que "[l]'écrivain qui compte, c'est celui qui a tout assimilé "(II,101), ou encore:

Comme lecteur, je suis aveuglé: tant de beauté, et tant de solitude dans tant de beauté, que j'en oublie où je dois aller, seulement conscient d'être bien en-dessous de ce texte sublime, ne désirant rien d'autre que de le manger, et de le manger éternellement puisque l'espoir désespéré de Melville je le fais mien. (III, 63)

Le second procédé que je voudrais analyser est celui du mimétisme et du dédoublement, qui dépasse celui-là le simple régime métaphorique pour devenir, au fil des pages, un enjeu narratif où se trouve cristallisée, en fait, toute l'américanité de

18 Victor-Lévy Beaulieu, Monsieur Melville. 1. Dans les aveilles de Moby Dick, Montréal, VLB éditeur, 1978, p. 23. L'ouvrage comprend deux autres tomes: 2. Lorsque souffle Moby Dick et 3. L'après Moby Dick ou la souveraine poésie. Pour les références subséquentes, j'indiquerai entre parenthèses, après chaque citation, la tomaison (en chiffres romains) et la pagination (en chiffres arabes). 
62

Monsieur Melville. Pour Abel Beauchemin, l'écriture du Melville ne se compare finalement en rien avec celle du Hugo ou du Kerouac: "Avec Melville, ça ne peut être que fort différent: ce que Melville a été, c'est ce que je voudrais être" (I, 23). Le projet littéraire d'Abel repose ainsi sur l'absolu du désir mimétique, qui devient la finalité même du processus anthropophage: "Il ne s'agit après tout que de parler de Melville et, l'espace du livre, de me substituer à lui" (I, 26). Certes, il faut admettre qu'au départ, Abel craint de dénaturer Melville: il associe ainsi la petite enfance de l'écrivain à "un lieu que je ne saurais retenir dans son temps et dans son espace, sinon en les faisant devenir miens, ce qui serait ne plus parler du tout de la même chose" (I, 68). Mais ses doutes se dissiperont progressivement, jusqu'à ce qu'il constate, au centre névralgique de l'œuvre, c'est-à-dire au milieu du deuxième tome, au moment où tout bascule, qu'il s'est transformé, littéralement, en Melville:

Je regarde cette main gauche qui, depuis trente heures, a écrit tant de feuillets, et je ne la reconnais plus: ma main est devenue la main même de Melville. Je passe les doigts dans mon visage, et je ne le reconnais plus: ma barbe est devenue la barbe même de Melville, tout comme mon nez, tout comme mes yeux qui se mettent à pleurer, tout comme mes cheveux extraordinairement épais, comme une suite de vagues se terminant dans le cou. (II, 157)

Devenu Herman Melville, le romancier Abel Beauchemin s'approprie non seulement son génie, mais aussi son bagage culturel, historique et géographique, pour entrer en possession de cette Amérique dont il rêvait, semble-t-il, depuis toujours. C'est à ce stade que se situe l'américanité réelle de Monsieur Melville: de la dévoration culturelle au mimétisme et à la substitution pure et simple, la voie est toute tracée. Le critique Pierre Nepveu résume d'ailleurs très bien cette américanité constitutive du roman de Beaulieu:

Ce n'est pas par hasard que le plus beau livre qui se soit écrit au Québec sur l'Amérique (au sens des États-Unis) soit le Melville de Victor-Lévy Beaulieu, livre où se dit, avec toute la passion et toute la lucidité propres à Beaulieu, cet immense désir mimétique qui nous travaille lorsque nous pensons "Amérique " et lorsque nous allons au-delà d'une vague référence au "tellurisme " de ce continent. ${ }^{19}$

19 Pierre Nepveu, "Paul-Marie Lapointe et la question de l'Améririue", Voix et images, $\mathrm{n}^{\circ} 51$, printemps 1992, p. 436. 
Le mimétisme me semble indissociable du dédoublement, c'est-à-dire du vaste réseau de correspondances qui est établi, tout au long de Monsieur Melville, entre l'univers américain et l'univers québécois dans lequel se débat Abel Beauchemin, de sorte qu'on ne parvient plus très bien, en dernier recours, à les distinguer. Il s'agit, encore une fois, d'un phénomène d'appropriation, qui caractérise au demeurant toute l'entreprise des Voyageries, suite romanesque qui gravite autour de l'univers des baleines et du golfe du Saint-Laurent - nouvel avatar de l'océan melvillien. Il est d'ailleurs intéressant de noter que le premier roman des Voyageries, Blanche forcée, s'ouvre sur deux phrases qui évoquent, mais en creux, le fameux "Call me Ishmael" de Moby Dick: “Comment je m'appelle, ç'a pas si tellement d'importance. Mettons Job J Jobin [...... 20. Quand, dans Monsieur Melville, Abel Beauchemin écrit la phrase mimétique par excellence, "Je m'appelle Herman Melville. Mettons" (II, 159), on peut constater toute la distance qui a été parcourue depuis Blanche forcée. Désormais, le dédoublement est permis, puisque la distance entre les deux écrivains se trouve abolie. Beaulieu peut dès lors se laisser porter par sa "passion des séries" (I, 100) et tenter de débusquer les multiples coïncidences qui le rapprochent de Melville: "Si Sartre se dédouble dans le personnage de Flaubert, et si moi qui écris ce livre j'en fais autant avec Melville, c'est que la passion des séries ne me quitte pas" (I, 100).

Le premier réseau de coincidences est d'ordre temporel ou historique. Beaulieu est sensible aux similarités qui caractérisent la littérature américaine à l'époque des commencements littéraires de Melville, soit dans les années 1840, et la littérature québécoise des années 1940, 1950 et même 1960. Quand Melville commence à écrire, la littérature américaine n'a pas encore le statut d'une littérature nationale, l'Angleterre exerçant toujours une forme de colonialisme culturel à l'endroit des États-Unis. Selon Beaulieu, "il faudra attendre la publication de la Lettre écarlate de Nathaniel Hawthorne pour que la littérature américaine prenne tout son sens et sa spécificité, dans les racines mêmes du pays [...]" (II, 70). Au contraire, comme elle a toujours été privée du soutien d'un projet collectif cohérent, la littérature québécoise serait demeurée, jusqu'aux années 1960 tout au moins, dans un état d'assujettissement:

20 Victor-Lévy Beaulieu, Blanche forcée, Montréal, VLB éditeur, 1976, p. 13. 
64

Je songe à la situation littéraire américaine telle qu'elle prévalait dans les années 1840 , et je me dis qu'elle ressemble en tous points à celle qui existait il y a encore peu de temps dans mon pays, les écrivains québécois attendant leur reconnaissance non pas de Montréal mais de Paris, rêvant dérisoirement d'écrire une langue qui, pour ressembler à la nôtre, ne pouvait toutefois dire totalement qui nous étions [...]. À cent ans de distance, nous revivions ce que Melville avait connu, courant après le mythe de nous-mêmes et nous imaginant le trouver dans une littérature qui ne tenait à peu près pas compte de notre point de vue particulier en Amérique. Il faudra attendre les années soixante pour que ce sentiment d'appartenance au continent américain se manifeste avec force et pour que nous comprenions que notre littérature, bien plus qu'avec les grands courants européens, avait partie liée avec la culture américaine. (II, 70-71)

On peut saisir, dès lors, la portée et la signification d'une entreprise comme celle de Monsieur Melville. En réintégrant le temps perdu de la vie et de l'écriture de Melville et en s'appropriant son discours, Beaulieu cherche à favoriser, au Québec, l'avènement simultané du mythe, du pays et d'une littérature véritablement nationale. Le projet littéraire d'Abel Beauchemin prévoit en effet que l'écriture du Melville va permettre celle de $L a$ grande tribu, qui est conçu comme le livre fondateur par excellence ou, pour reprendre l'expression déjà citée de Beaulieu, "l'œuvre abolissant toutes les autres parce que, d'un seul coup, donnant tous leurs sens aux autres" 21 . Pour y arriver, il lui faut absolument dévorer le prototype des écrivains américains, ce Melville obsédant qu'il en vient à piller sans vergogne, en dépit de la colère de son frère Jos Connaissant qui l'accuse, non seulement de vouloir se "donner du talent", mais aussi d'être "un imposteur, doublé d'un usurpateur" (II, 182). Ce à quoi Abel répond:

Moi, je peux peut-être démonter le mécanisme et le recomposer, mais je ne peux pas l'inventer. C'est ce qui fait que, face à Melville, je me comporte en usurpateur et en imposteur qui ne cherche qu'à se l'approprier et qu'à monter dessus pour oublier jusqu'à quel point tout, en moi, vole bas. (II, 182)

Le deuxième réseau de coïncidences est de nature spatiale ou géographique. Dans le but de dégager les multiples correspon-

21 Consulter à ce sujet Marie-Josée Blais et Jean Morency, "L'béritage de Victor-Lévy Beaulieu: un "testament" littéraire?:, Dalbousic Frencb Studies, $\mathrm{n}^{\circ} 23$, automne-hiver 1992, p. 97-104. 
dances entre son univers et celui de Melville, Beaulieu va effectuer plusieurs rapprochements entre le paysage québécois et celui de la Nouvelle-Angleterre, notamment entre les hauteurs de la Mattavinie, dans le nord du comté de Lanaudière, et les collines des Berkshires, dans le Massachusets, où a vécu Melville. Abel Beauchemin remarque ainsi que la Mattavinie "ressemble étrangement au Massachusets de Melville" (I, 150):

Même configuration du sol, mêmes digues de cailloux en guise de clôtures, mêmes sapins et mêmes épinettes trapus à cause de tout ce roc sous les quelques pouces de terre meuble. Après tout, ce n'est pas pour rien que Melville et moi, nous nous entendons bien: nous partageons le même monde, nous sommes du même côté des choses. (I,150)

Par conséquent, ce n'est pas uniquement l'acte d'écriture qui rapproche Abel de Melville, mais le décor américain dans lequel ils évoluent tous les deux, hérité de la patience des pionniers et de "ce qu'il y a de désespéré dans le courage" (I, 150). Pour poursuivre la rédaction du Melville, Abel ira même jusqu'à prendre la direction de la Mattavinie, dans le but d'abolir la distance qui le sépare de l'auteur de Moby Dick:

C'est tout à fait comme j'avais imaginé que ça serait: la distance entre la Mattavinie et les Berkshires s'est estompée presque miraculeusement dès que la vieille cadillac couleur rouge sang, avec de grands ailerons lumineux, s'est retrouvée dans les Hauts de Sainte-Emmilie. (II, 223)

Sur le chemin du mimétisme et du dédoublement, il reste toutefois un obstacle de taille dont j'aimerais maintenant dire quelques mots. Il s'agit de la langue de Melville, dont l'étrangeté s'avère finalement beaucoup plus radicale que celle de l'écrivain ou de l'histoire et des paysages américains. Beaulieu attend au troisième tome de Monsieur Melville pour avouer qu'il ne sait pas bien lire l'anglais (III, 49) et qu'il a été souvent dans l'obligation de recourir à des traductions pour saisir l'univers imaginaire de Melville. Les extraits des romans de Melville sont d'ailleurs toujours cités dans leur traduction française. Mais au moment d'entreprendre l'étude de certains contes et des poèmes de Melville, qui n'étaient pas encore traduits en français en 1977, Beaulieu doit recourir aux services de Michel Garneau, dont on connait par ailleurs la fameuse adaptation, en langue "québécoise", du Macbetb de Shakespeare. Néanmoins, dans Monsieur Melville, c'est l'amérindienne Samm qui guidera Abel au cour de l'univers poétique de l'écrivain américain: 
66

D'où l'importance de Samm à mes côtés: étant d'origine amérindienne, donc doublement plus aliénée que moi qui ne suis que québécois et unilingue, elle connaît tous les langages. C'est pourquoi, tout le temps de mon livre, je veux la garder muette, ne lui confiant que le pire des travaux: me traduire ce que je ne comprends pas des contes de Melville pour que je puisse me vérifier dans mes intuitions. (III, 51)

Cette question de la langue et de la traduction pose en fait plusieurs problèmes au désir mimétique et anthropophage à l'œuvre dans Monsieur Melville. Car comment faire sien un discours qui, dans son code linguistique même, vous demeurera toujours irréductiblement étranger, sinon en le traduisant, mais cette fois au risque de le dénaturer? Selon Annie Brisset, qui a étudié le problème dans Sociocritique de la traduction, "le texte étranger est "naturalisé" par la traduction qui l'introduit dans une société nouvelle"22, ce qui est d'autant plus vrai sur le marché québécois des biens symboliques, dans lequel "l'étranger est une anti-valeur»23. En traduisant la poésie de Melville, Beaulieu et Garneau la "naturalisent " et l'intègrent au projet national québécois, un peu comme Garneau l'avait fait pour Shakespeare. Dans Monsieur Melville, on ne trouve en réalité que trois courts poèmes qui n'ont pas été traduits, parce qu'ils sont, selon Abel, "au delà de toute beauté * (III, 202); par conséquent, leur altérité est surtout d'ordre esthétique, ce que le recours au texte original ne fait que souligner. Dans tous les autres cas, le discours de Melville est assimilé et expurgé de son étrangeté. Il se trouve donc, littéralement, avalé par le discours de Beaulieu. Je cite à ce propos Annie Brisset:

Dans la société québécoise encore peu certaine de son identité, la traduction remplit d'abord une fonction spéculaire de nature à légitimer le discours constitutif de la représentation sociale, en lui apportant de l'extérieur des appuis qui le confortent [...]. Dans une société où la littérature a partie liée avec le politique, où l'on proclame que l'existence même de la "nation québécoise" est subordonnée à celle d'une littérature propre, le travail de traduction recoupe celui de l'écriture: consolider les idéologèmes de la représentation sociale, notamment dans ses dimensions historiques et politiques. Cela même implique une mise entre

\footnotetext{
22 Annie Brisset, Sociocritique de la traduction. Théâtre et altérité au Québec (1968-1988), Longueuil, Le Préambule, coll. "L'univers des discours", 1990, p. 26.

23 Ibid., p. 70.
} 
parenthèses de l'altérité fondamentale du texte étranger, appelé à cautionner la représentation du fait québécois. ${ }^{24}$

Dès lors, on peut saisir, il me semble, toute l'importance de l'intertexte américain dans la littérature contemporaine au Québec, ainsi que la place du discours sur l'américanité dans le domaine de la critique littéraire. Le Québec des années 1970 et 1980 cherche non seulement à maintenir "le relais français", pour reprendre l'expression de François Ricard, mais aussi à découvrir ou à redécouvrir le "relais américain", dans une dévoration culturelle qui se fait tous azimuts. Dans cette perspective, l'étude d'un roman comme Les grandes marées de Jacques Poulin, qui est paru la même année que Monsieur Melville, pourrait nous renseigner plus avant sur le recours aux textes littéraires américains et sur la question de la traduction, le protagoniste du roman de Poulin étant d'ailleurs un traducteur de bandes dessinées américaines... De manière encore plus large, on pourrait voir, dans le processus de l'intégration et du croisement des discours étrangers dans la littérature québécoise contemporaine, l'indice d'une situation de crise, soit identitaire, soit nationale, que viendraient résoudre les emprunts au domaine étranger. Selon Laurent Jenny, l'usage intertextuel des discours constitue "l'instrument de parole privilégié des époques d'effritement et de renaissance culturels " ${ }^{25}$. Au fond, le débat autour de la francité et de l'américanité de la littérature et de la culture québécoises ne fait peut-être que démontrer que nous ne sommes encore, en tant que peuple, que peu de choses. Le Melville de Victor-Lévy Beaulieu devient alors la simple manifestation d'une catastrophe se déroulant sur fond de vide, et ne débouchant sur rien d'autre que de la béance. Parvenu à ce stade, je laisserai donc Beaulieu conclure:

Je pense que ce Monsieur Melville que j'ai écrit n'a peut-être de sens que par rapport à ce malentendu - je suis Québécois et, pendant tout le temps de l'écriture de Monsieur Melville, je n'ai existé qu'en fonction de l'autre, c'est-à-dire cet écrivain américain sur le dos duquel je me suis fixé comme un pou de baleine parce que je n'avais pas le choix de mon malentendu être Québécois, c'est n'avoir ni temps ni espace, c'est vivre dans le creux d'une béance historique, c'est ne pas être au monde sinon en se colletaillant avec son absurde - par exemple, moi écrivant non pas un essai ni même une biographie de Melville,

24 Brisset, op. cit., p. 252-253.

25 Jenny, op. cit., p. 281. 
68

ce qui aurait été historique, mais rédigeant une lecture-fiction pour me démontrer à moi-même, en tant que Québécois, que si je suis, ce ne peut-être encore que par personne interposée, que par écriture interposée [...]. ${ }^{26}$

26 Beaulieu, Entre la sainteté et le terrorisme, p. 463. 